\title{
Full-Color LCD Microdisplay System Based on OLED Backlight Unit and Field-Sequential Color Driving Method
}

\author{
Sungho Woo, ${ }^{1}$ Dae-Hwan Kim, ${ }^{1}$ Yoon Soo Han, ${ }^{2}$ and Byeong-Dae Choi ${ }^{3}$ \\ ${ }^{1}$ Green Energy Research Division, Daegu Gyeongbuk Institute of Science and Technology (DGIST), 50-1 Sang-ri, Hyeonpung-myeon, \\ Dalseong-gun, Daegu 711-873, Republic of Korea \\ ${ }^{2}$ Department of Advanced Energy Material Science and Engineering, Catholic University of Daegu, Geumrakro 5, Hayang-eup, \\ Gyeongsan-si, Gyeongbuk 712-702, Republic of Korea \\ ${ }^{3}$ Division of Nano and Bio Technology, Daegu Gyeongbuk Institute of Science and Technology (DGIST), 50-1 Sang-ri, \\ Hyeonpung-myeon, Dalseong-gun, Daegu 711-873, Republic of Korea
}

Correspondence should be addressed to Byeong-Dae Choi, bdchoil@dgist.ac.kr

Received 30 May 2011; Accepted 27 July 2011

Academic Editor: Gion Calzaferri

Copyright ( 2011 Sungho Woo et al. This is an open access article distributed under the Creative Commons Attribution License, which permits unrestricted use, distribution, and reproduction in any medium, provided the original work is properly cited.

\begin{abstract}
We developed a single-panel LCD microdisplay system using a field-sequential color (FSC) driving method and an organic lightemitting diode (OLED) as a backlight unit (BLU). The $0.76^{\prime \prime}$ OLED BLU with red, green, and blue (RGB) colors was fabricated by a conventional UV photolithography patterning process and by vacuum deposition of small molecule organic layers. The fieldsequential driving frequency was set to $255 \mathrm{~Hz}$ to allow each of the RGB colors to be generated without color mixing at the given display frame rate. A prototype FSC LCD microdisplay system consisting of a $0.7^{\prime \prime}$ LCD microdisplay panel and the $0.76^{\prime \prime}$ OLED BLU successfully exhibited color display and moving picture images using the FSC driving method.
\end{abstract}

\section{Introduction}

Organic light-emitting diodes (OLEDs) have attracted extensive attention during the past two decades for potential applications in flat panel displays owing to their intrinsic properties such as self emission, low weight, high luminance, high contrast, wide viewing angle, and fast response speed [1-3]. The performance and stability of OLEDs have rapidly improved over the last few years, leading to their utilization in commercial applications such as small area displays and general lighting devices. However, their market share in the display industry has not increased as fast as expected because of the difficulty in increasing the display sizes with uniform luminance driven by the active matrix scheme [4].

In order to expand OLED market, it is worth noting the case of light emitting diode (LED) industry. The demand for high-performance LEDs has increased greatly with the emergence of new technologies and applications such as signage, automotive lighting, mobile display devices, and LED backlight units (BLUs) for LED TVs. From this point of view, our group has been attempting to develop OLEDs as BLUs for LCD microdisplays [5].
Microdisplays with a diagonal size of less than $1^{\prime \prime}$ $(25.4 \mathrm{~mm})$ are widely used not only in small screen devices such as camcorder viewfinders, digital cameras, and other portable devices but also in large screen devices such as projectors, TVs, head-mounted displays (HMD), and 3D displays $[6,7]$.

However, full-color microdisplays may have some limitations when used in LCD or OLED devices, because of reduction in the pixel size, such as a decrease in the luminance efficiency and production yield with a decrease in the aperture ratio. In order to resolve these problems, we have developed an LCD microdisplay based on the OLED BLU and that uses a field-sequential color (FSC) driving method.

A full-color display can be achieved using the FSC driving method wherein, three monochromatic images corresponding to the three primary colors, red (R), green $(\mathrm{G})$ and blue (B) are combined in a repetitive sequence and at a frame rate that is more than three times that of the conventional frame rate $(60 \mathrm{~Hz})[8]$. This eliminates the need for subpixels and color filter layers and provides a high transmittivity, a large aperture ratio, and the possibility of increasing the pixel density by at least three times [8-10]. 
[ Top view ]

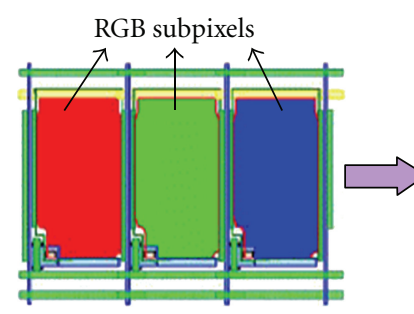

[ Side view ]

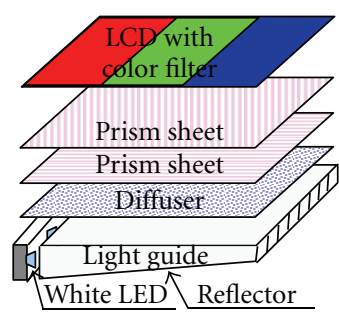

(a)

(b)

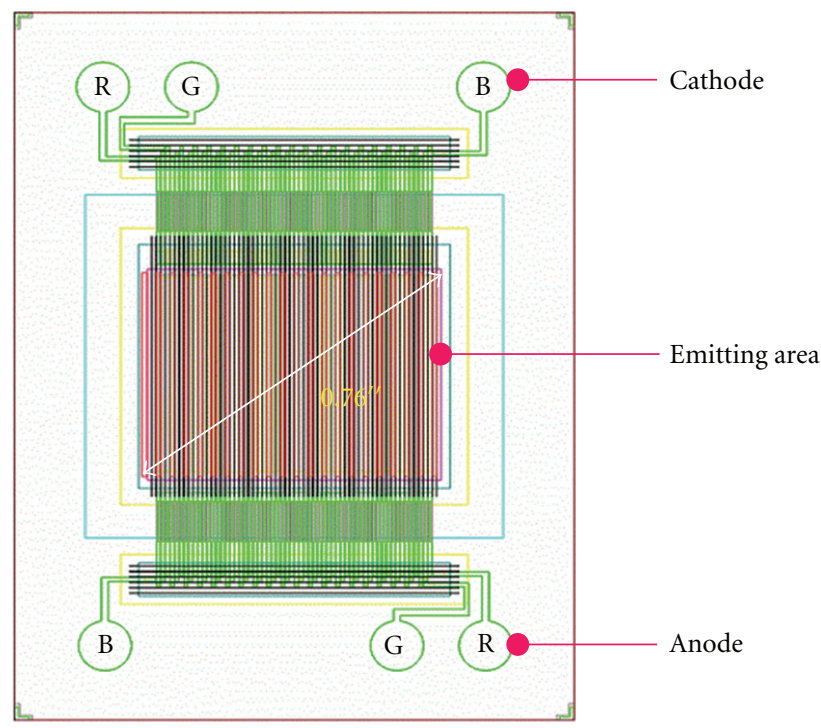

(a)

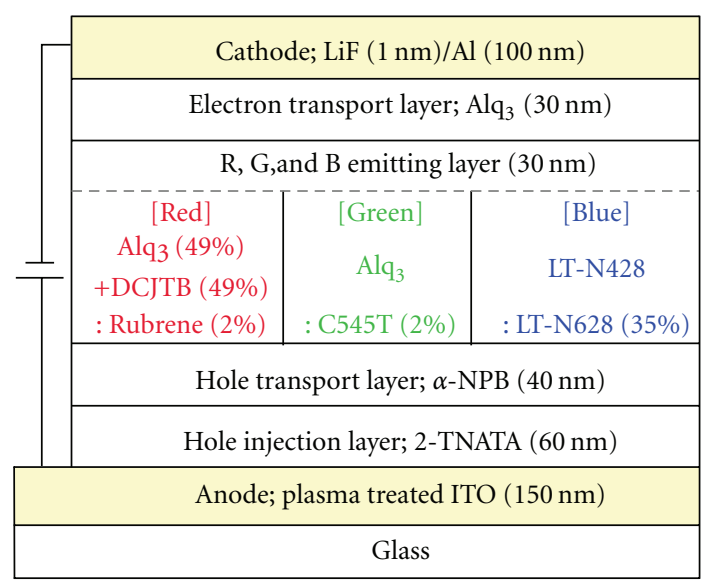

(b)

FIgURE 2: Structure of stripe-patterned RGB OLED device; (a) Top (b) Cross-sectional view. ratio, and large pixel density over conventional ones.

In this paper, we describe in detail the development of a single-panel LCD microdisplay system using an OLED BLU and the FSC driving method in terms of the optimization of the aperture ratio as well as the electro-optical and image display performances of the microdisplay.

\section{Experimental}

2.1. Materials and Fabrication of OLED BLU. The $0.76^{\prime \prime}$ OLED BLU with high aperture ratio (73\%), which has stripepatterned RGB arrays, was fabricated using conventional materials and methods as shown in Figure 2(a). The OLED BLU was made on a patterned ITO $(150 \mathrm{~nm}, 15 \mathrm{ohm} / \square)$ glass substrate integrated with a well-defined insulating layer and a cathode separator in order to separate the RGB colors. Its layer structure is shown in Figure 2(b).

Materials: 4, $4^{\prime}, 4^{\prime \prime}$-Tris (2-naphthylphenylamino) triphenylamine [2-TNATA] was purchased from E.L.M. Co., Ltd., Korea. N, $\mathrm{N}^{\prime}$-Di-(naphthalene-1-yl)-N, $\mathrm{N}^{\prime}$-diphenyl- benzidine $[\alpha$-NPB], 10-(2-benzothiazolyl)-1,1,7,7-tetramethyl2,3,6,7-tetrahydro- $1 \mathrm{H}, 5 \mathrm{H}, 11 \mathrm{H}$-(1) benzopyrano $(6,7,8$ ij)-quinolizin-11-one [C545T], and 4-(dicyanomethylene)2-tert-butyl-6-(1,1,7,7-tetramethyljulolidyl-9-enyl)-4H-pyran [DCJTB] were purchased from Sun Fine Chem Co., Ltd., Korea. Tris(8-hydroxyquinolinato) aluminum [Alq ${ }_{3}$, $(5,6,11,12)$-tetraphenylnaphthacene [Rubrene], 2,2' -Di-pyrenyl-9,9-spirobifluorene [LT-N428], and [LT-N628] were obtained from Lumtec Co., Ltd., Taiwan.

All the organic materials were used in as received state without further purification. The organic layers and metal electrode layers were formed using a vacuum thermal evaporator $\left(5.0 \times 10^{-6}\right.$ Torr $)$ equipped with a CCD alignment system and a metal shadow mask. After the cathode electrode deposition, we encapsulated the OLED device using cover glass and getter material to protect the device from deterioration by oxygen and moisture. 


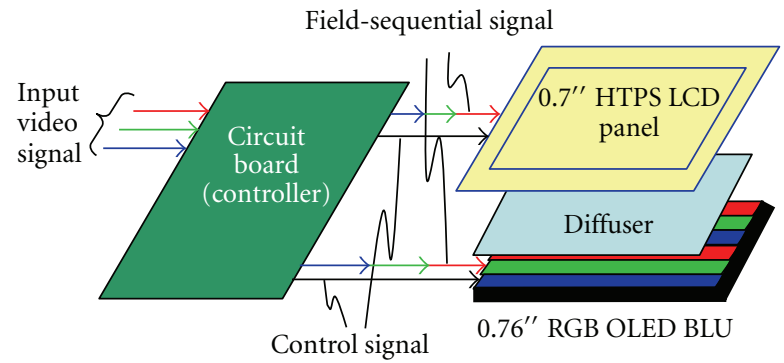

(a)

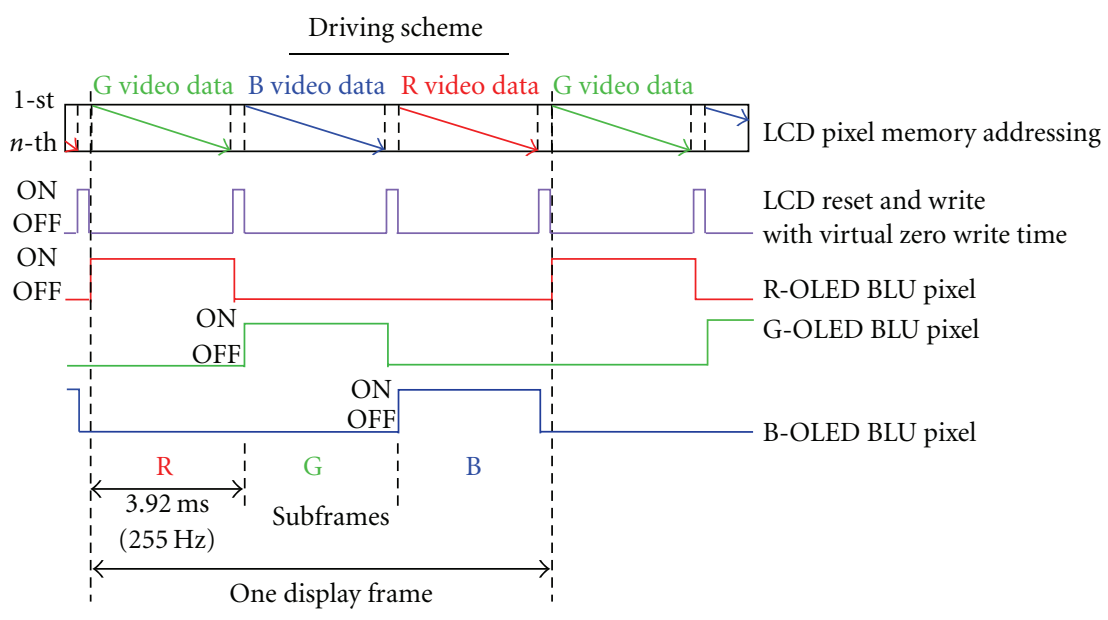

(b)

FIgURE 3: (a) Configuration of FSC LCD microdisplay system based on OLED BLU and (b) FSC driving scheme.

2.2. Organization of LCD Microdisplay System. As shown in Figure 3(a), the LCD microdisplay system consists of a $0.7^{\prime \prime}$ SVGA high-temperature polysilicon (HTPS) LCD panel (with $800 \times 600$ resolution and a $67 \%$ aperture ratio, from ILJIN Display Co., Ltd., Korea), control board, and $0.76^{\prime \prime}$ RGB OLED BLU. The FSC driving scheme is shown in Figure 3(b). We chose a fast driving frequency, more than $180 \mathrm{~Hz}$, which is three times the conventional frame rate, to eliminate color mixing or color breakup. One display frame is divided into three subframes of $3.92 \mathrm{~ms}(255 \mathrm{~Hz})$ to represent the red, green, and blue images sequentially. In order to accommodate this high rate of FSC driving, we used an LCD panel which contains a twisted nematic (TN)-LC with a high refractive index anisotropy $(\Delta n)$ and reduced cell gap of about $2 \mu \mathrm{m}$ to improve its response time to $1.8 \mathrm{~ms}$ including rising time of $1.5 \mathrm{~ms}$ and falling time of $0.3 \mathrm{~ms}$ as described in the patent of ILJIN Display Co. [11]. Moreover, the LCD adopts a pixel memory addressing scheme to realize the virtual zero write time as indicated in Figure 3(b). To achieve this driving scheme, the LC pixel is composed of two capacitors such as a video data storage capacitor $\left(C_{S}\right)$ and an LC writing capacitor $\left(C_{W}\right)$ and three thin film transistors (TFTs) such as a data addressing TFT $\left(M_{A}\right)$, an LC writing TFT $\left(M_{W}\right)$, and an LC reset TFT $\left(M_{R}\right)$. (The pixel circuit configuration is not shown here.)

During the first subframe, previously addressed red LC pixel is on, and the red OLED BLU is simultaneously illuminated, while the green video data for second subframe is applied and stored through the $M_{A}$ and the $C_{S}$, and then, LC reset and write pulse are applied to the $M_{R}$ and the $M_{W}$ to delete previous data and transfer the green video data from the $C_{S}$ to the $C_{W}$ during virtually zero write time. (More detailed information about this pixel memory addressing scheme is described in the patent of ILJIN Display Co. [12].) By using a pixel memory addressing scheme, it is possible to separate the LC addressing time and the BLU illumination time. Considering this driving scheme, response time of $1.8 \mathrm{~ms}$ is enough to drive LC within one subframe of $3.92 \mathrm{~ms}$, while we can illuminate BLU nearly full time of subframe.

2.3. Characterization of FSC OLED BLU System. We measured the current density-voltage-luminance (J-V-L) and emitting spectrum properties using a CS1000 Spectroradiometer and a Keithley 236 Source Meter. The electrooptical response property of the FSC driving OLED BLU was investigated by a digital oscilloscope (Tektronix, DPO-4104) and a photodiode with a power meter (Newport).

\section{Results and Discussion}

3.1. Optimization of Aperture Ratio (Stripe Pattern Interval) in OLED Design. Prior to making a full-color OLED device, we tested four kinds of devices with an aperture ratio of $56 \%, 67 \%, 73 \%$, and $81 \%$, which is the ratio of emitting area to nonemitting area in one pixel, and found the optimum aperture ratio for high brightness without traces of stripe 


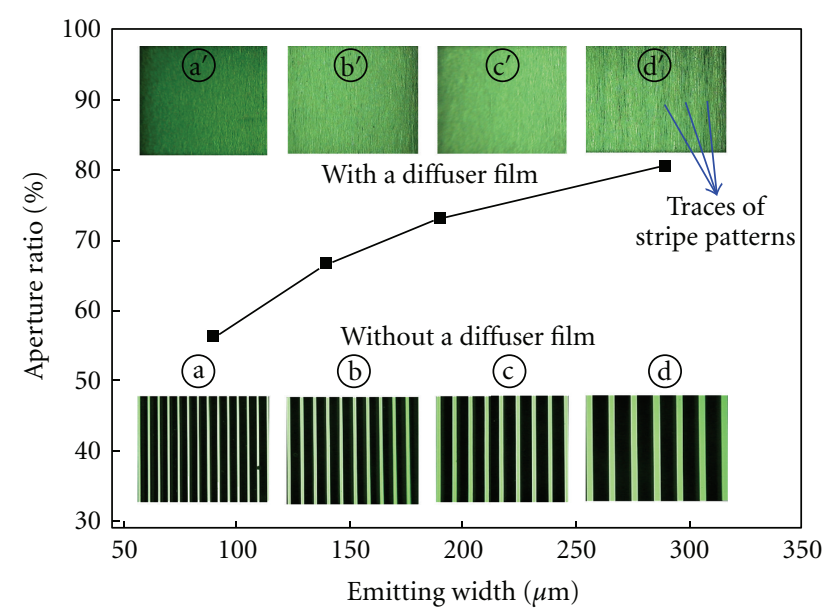

FIgURE 4: Emitting images as function of emitting width (or aperture ratio) with and without diffuser film (the aperture ratio and emitting width are $56 \%$ and $90 \mu \mathrm{m}$ for (a), $67 \%$ and $140 \mu \mathrm{m}$ for (b), $73 \%$ and $190 \mu \mathrm{m}$ for (c), and $81 \%$ and $290 \mu \mathrm{m}$ for (d), respectively).

patterns when the OLED is covered with a thin diffuser film. The pitches in Figure 4 are $480 \mu \mathrm{m}, 630 \mu \mathrm{m}, 780 \mu \mathrm{m}$, and $1080 \mu \mathrm{m}$ corresponding to aperture ratio of $56 \%, 67 \%, 73 \%$, and $81 \%$. Considering the full-color array structure, we take one-third of pitch size as a real pitch size of each R, G, and B pixel when calculate the aperture ratio. As the aperture ratio increases, both the width of the emitting area of one pixel and the interval between the emitting areas increase. Although the aperture ratio of $81 \%$ had the highest brightness, we selected the aperture ratio of $73 \%$ in our OLED design because of the lack of stripe patterns at this aperture ratio (see Figure 4).

3.2. Electro-Optical Performances of OLED BLU. The emitting images of $\mathrm{R}, \mathrm{G}, \mathrm{B}$, and $\mathrm{W}$ with and without diffuser film for device with an aperture ratio of $73 \%$, and their emitting spectra were shown in Figure 5.

The emitting images with diffuser film showed clear R, G, B, and W colors without any stripe patterns though having some defects. Their emitting spectrum had three peaks at $623 \mathrm{~nm}, 519 \mathrm{~nm}$, and $460 \mathrm{~nm}$. Our red, green, and blue emitting layers were host-dopant systems of fluorescent material designed to improve the luminous efficiency [13, 14]. The red emitter with DCJTB doped into the $\mathrm{Alq}_{3}$ : rubrene mixed-host showed a strong red emission with negligible EL emission from the $\mathrm{Alq}_{3}(524 \mathrm{~nm})$, which means a complete energy transfer from Alq ${ }_{3}$ to DCJTB. It is well known that rubrene may play an intermediate role for the cascade energy transfer process from $\mathrm{Alq}_{3}$ to DCJTB, because the HOMO and LUMO of rubrene exist between those of $\mathrm{Alq}_{3}$ and DCJTB in the red emitting system [13-16]. We can also see the complete emission spectra of green and blue in the plot, but the blue spectrum has a weak tail peak in the green region that could have originated from crosstalk between the green and blue pixels because of an insignificant electrical short in the device.

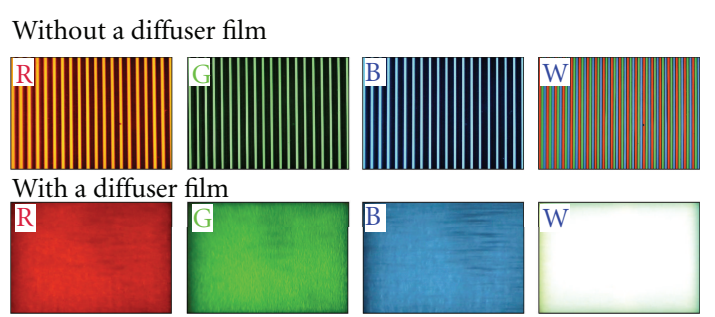

(a)

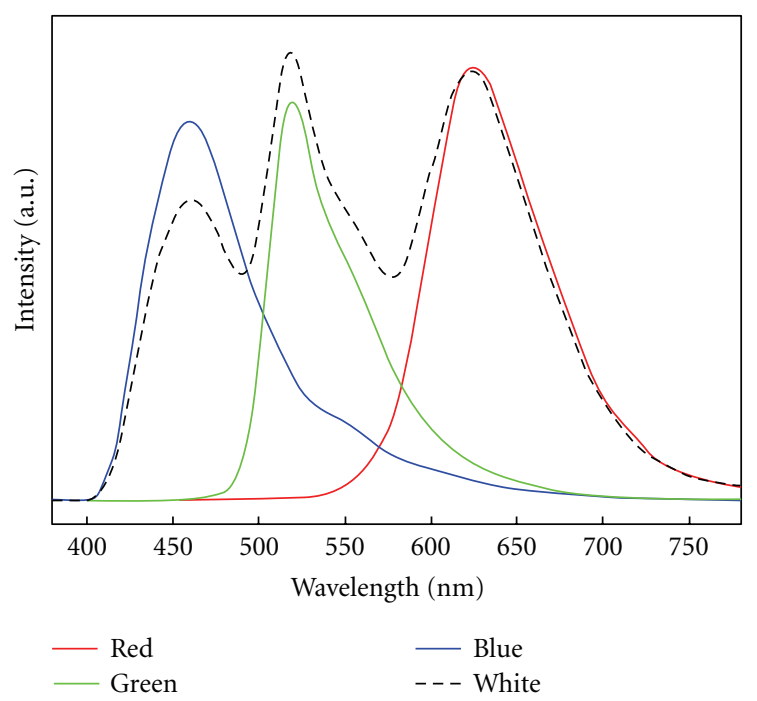

(b)

FIGURE 5: (a) Emitting image of R, G, B, and W with and without diffuser film and (b) emitting spectrum of fabricated RGB stripepatterned OLED device.

Figure 6 shows the V-L curve of the full white state OLED device without the diffuser sheet. The OLED device turned on at a voltage of $3.5 \mathrm{~V}$, and the white luminance under an applied voltage of $10 \mathrm{~V}$ was $500 \mathrm{~cd} / \mathrm{m}^{2}$. Although the emitting performance of our OLED device was a little lower compared to state-of-the-art OLED devices because of the use of low-efficiency fluorescent emitting materials in our device, we think that there is much room for improvement in our system, by applying newly developed materials such as a phosphorescent emitting materials and high-efficiency charge injection materials [17-19].

Figure 7 shows the electro-optical response of the FSC driven OLED BLU at a frequency of $255 \mathrm{~Hz}$. OLEDs are generally very fast devices with on/off response times in the microsecond range. The RGB pixels of the OLED BLU emitted successfully according to their subframe duration $(3.92 \mathrm{~ms})$. This means that our OLED device is sufficiently fast for an FSC-driven BLU.

3.3. Image Display Properties. Figure 8 shows a full-color display image of our $0.7^{\prime \prime}$ prototype FSC LCD micro display system. The displayed images were indeed clear moving pictures, and there was no color breakup or color mixing due to the fast response properties of both the OLED BLU 


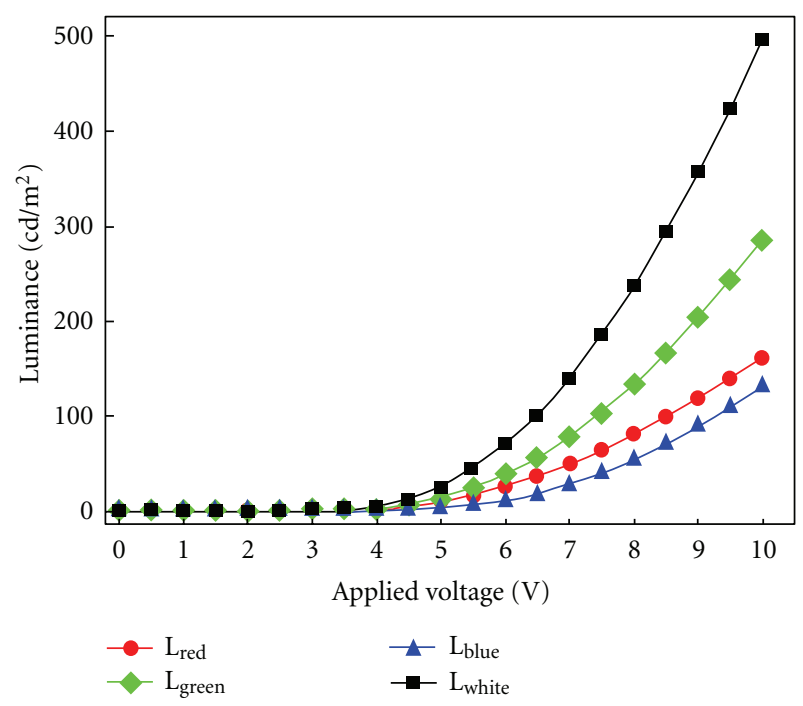

FIGURE 6: Luminance as function of applied voltages for R, G, B, and $\mathrm{W}$ state OLED.

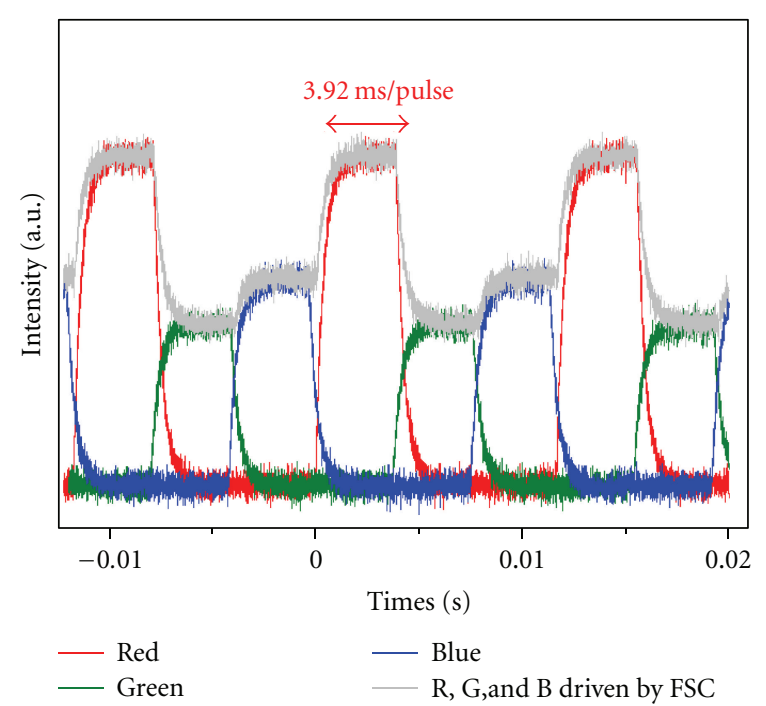

FIgURE 7: Electro-optical response of FSC driving OLED BLU.

$(255 \mathrm{~Hz}$ frame rate) and the LCD panel (1.8 $\mathrm{ms}$ response time).

Our OLED BLU and LCD panel have potential for faster driving performance, so we are now trying to develop a 3D display employing an additional cylindrical microlens array (MLA) attached to our newly developed microdisplay system.

\section{Conclusions}

We have developed a single-panel LCD microdisplay system using a newly designed RGB color OLED device as a BLU. A low-cost microdisplay system with high optical efficiency and high pixel density was realized using the OLED BLU and the FSC driving method, as they eliminate the need for color filter layers, subpixels, and various optical films. Our

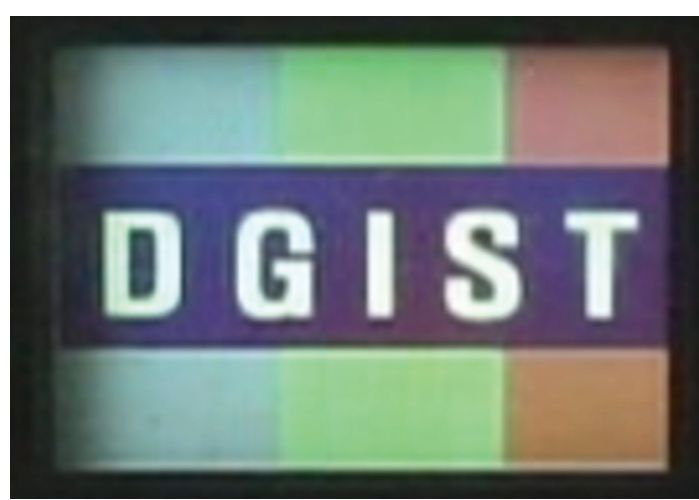

(a)

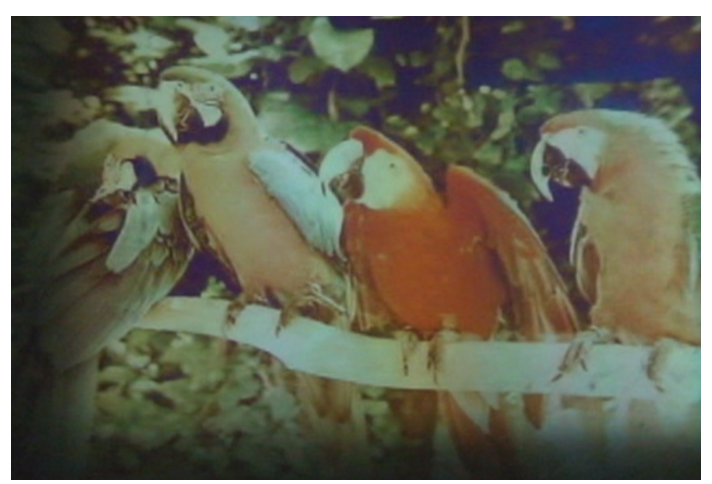

(b)

FIguRE 8: Captured images of color display operation of $0.7^{\prime \prime}$ microdisplay system.

microdisplay system shows a fast response speed, distinct color display, and clear moving picture images. The proposed microdisplay can open up a new application area of OLED devices as BLUs. It might be useful in a wide range of applications from small size mobile displays (microprojector, head-up display, mobile phone, etc.) to flexible large size displays by introducing a flexible LCD panel and a flexible OLED BLU. We also expect the development of 3D display systems using our microdisplay with an additional MLA in the near future.

\section{Acknowledgments}

This work was supported by the DGIST Basic R\&D Program of the Ministry of Education, Science, and Technology (MEST) of Korea. The authors would like to express thanks to Mr. Kil Whan Oh (ILJIN DISPLAY Co.) for his fruitful discussions on the $0.7^{\prime \prime}$ SVGA HTPS LCD panel, Ms. Gwi Jeong Cho (Kyungpook National University) for assisting OLED fabrication processes, and Ms. Yun Seon Do (DGIST) for helping the measurement of electro-optical response property. The authors also would like to give a special thanks to Professor Youngkyoo Kim of Kyungpook National University for his great advice on OLED devices. 


\section{References}

[1] Y. Kim and C. S. Ha, Advances in Organic Light-Emitting Device, Trans Tech Publications Ltd, Switzerland, 2008.

[2] A. Misra, P. Kumar, M. N. Kamalasanan, and S. Chandra, "White organic LEDs and their recent advancements," Semiconductor Science and Technology, vol. 21, no. 7, article R01, pp. R35-R47, 2006.

[3] K. Y. Peng, C. C. Lin, C. T. Lee, and C. I. Wu, "Enhanced holeinjection in organic light-emitting devices by utilizing F4TCNQ and the interface analysis by ultraviolet photoelectron spectroscopy," in Proceedings of the 13th International Display Workshops (IDW'06), pp. 1279-1281, December 2006.

[4] M. Eritt, C. May, K. Leo, M. Toerker, and C. Radehaus, "OLED manufacturing for large area lighting applications," Thin Solid Films, vol. 518, no. 11, pp. 3042-3045, 2010.

[5] S. H. Woo, D. H. Kim, Y. S. Do, Y. S. Han, and B. D. Choi, "Microdisplay system using field-sequential color LCD with 3-primary colors OLED backlight," in Proceedings of the 14th International Display Workshops (IDW '07), pp. 1041-1043, December 2007.

[6] O. Prache, "Active matrix molecular OLED microdisplays," Displays, vol. 22, no. 2, pp. 49-56, 2001.

[7] F. Su, Y. Sun, C. Y. Audrey Wong, H. L. Ho, and X. Chen, "Development of an integrated optical system for warpage and hermeticity test of microdisplay," Optics and Lasers in Engineering, vol. 45, no. 12, pp. 1177-1185, 2007.

[8] K. I. Takatori, "Field-sequential smectic LCD with twin-gateTFT pixel amplifiers," Displays, vol. 25, no. 1, pp. 37-44, 2004.

[9] K. Käläntär, T. Kishimoto, K. Seklya, T. Miyashita, and T. Uchida, "Spatio-temporal scanning backlight for color-field sequential optically compensated bend liquid-crystal display," in Proceedings of the SID International Symposium, pp. 13161319, May 2005.

[10] Y. Fuiisaki, H. Sato, Y. Inoue et al., "Field-sequential color LCD panel driven by low-voltage operation organic TFT," in Proceedings of the 13th International Display Workshops, IDW '06, pp. 1687-1690, December 2006.

[11] K. H. Hur, J. W. Bae, and H. H. Jang, Liquid Crystal Display Device WO/2007/111402, 2007.

[12] K. S. Park and K. H. Oh, Liquid Crystal Display WO/2008/039009, 2008.

[13] T. H. Liu, C. Y. Iou, and C. H. Chen, "Doped red organic electroluminescent devices based on a cohost emitter system," Applied Physics Letters, vol. 83, no. 25, pp. 5241-5243, 2003.

[14] H. Kanno, Y. Hamada, and H. Takahashi, "Development of OLED with high stability and luminance efficiency by codoping methods for full color displays," IEEE Journal on Selected Topics in Quantum Electronics, vol. 10, no. 1, pp. 3036, 2004.

[15] H. Y. Kang, G. W. Kang, K. M. Park, I. S. Yoo, and C. Lee, "Comparison of the electroluminescence of a red fluorescent dye doped into the $\mathrm{Alq}_{3}$ and $\mathrm{Alq}_{3}$ : rubrene mixed host," Materials Science and Engineering C, vol. 24, no. 1-2, pp. 229232, 2004

[16] Y. Hamada, H. Kanno, T. Tsujioka, H. Takahashi, and T. Usuki, "Red organic light-emitting diodes using an emitting assist dopant," Applied Physics Letters, vol. 75, no. 12, pp. 1682-1684, 1999.

[17] L. S. Hung and C. H. Chen, "Recent progress of molecular organic electroluminescent materials and devices," Materials Science and Engineering R: Reports, vol. 39, no. 5-6, pp. 143222, 2002.
[18] S. Kappaun, C. Slugovc, and E. J. W. List, "Phosphorescent organic light-emitting devices: working principle and iridium based emitter materials," International Journal of Molecular Sciences, vol. 9, no. 8, pp. 1527-1547, 2008.

[19] K. T. Kamtekar, A. P. Monkman, and M. R. Bryce, "Recent advances in white organic light-emitting materials and devices (WOLEDS)," Advanced Materials, vol. 22, no. 5, pp. 572-582, 2010. 


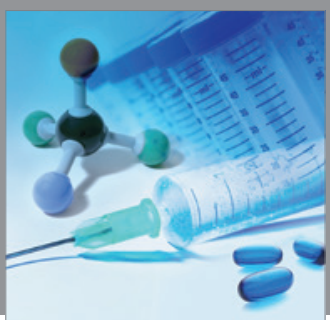

International Journal of

Medicinal Chemistry

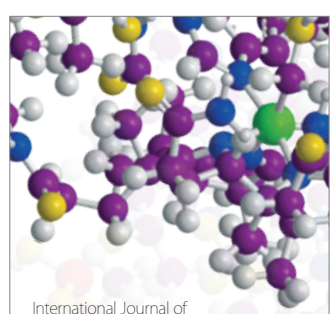

Carbohydrate Chemistry

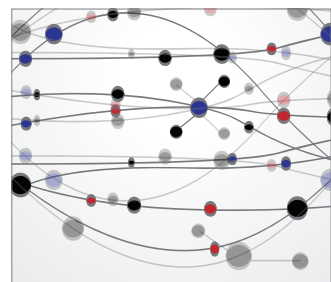

The Scientific World Journal
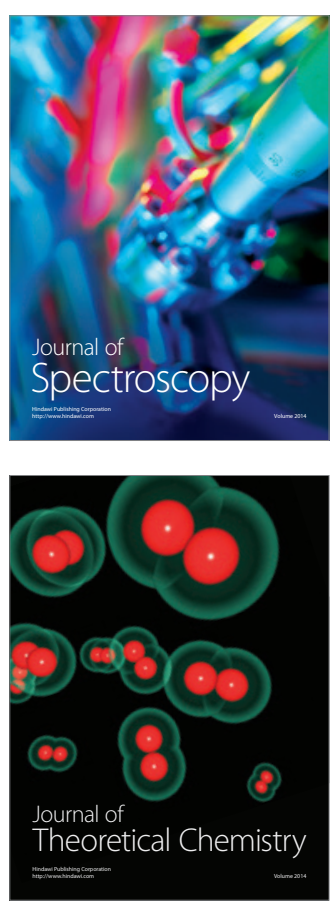
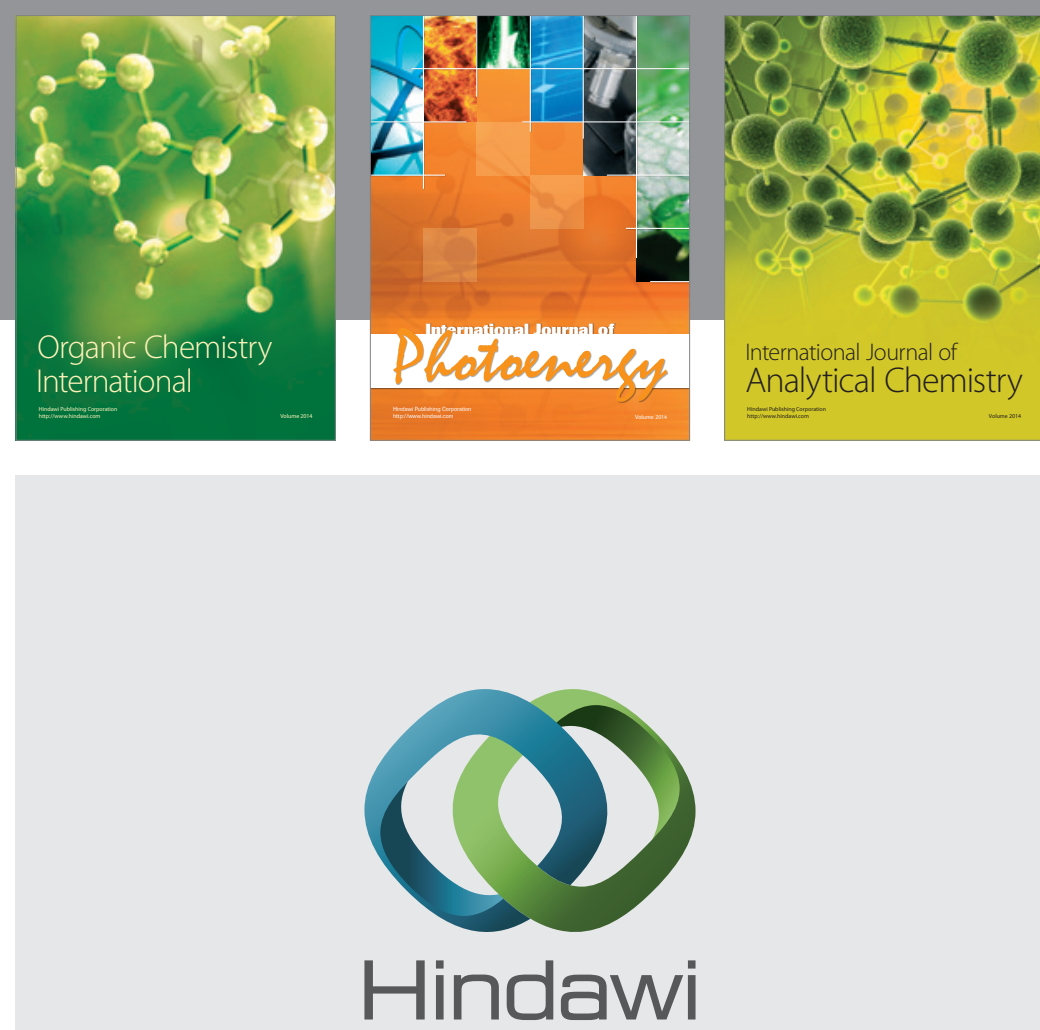

Submit your manuscripts at

http://www.hindawi.com
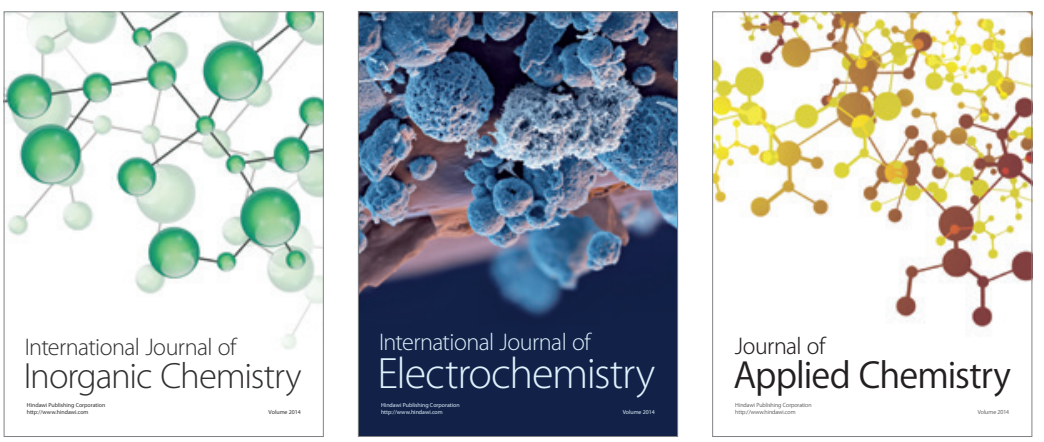

Journal of

Applied Chemistry
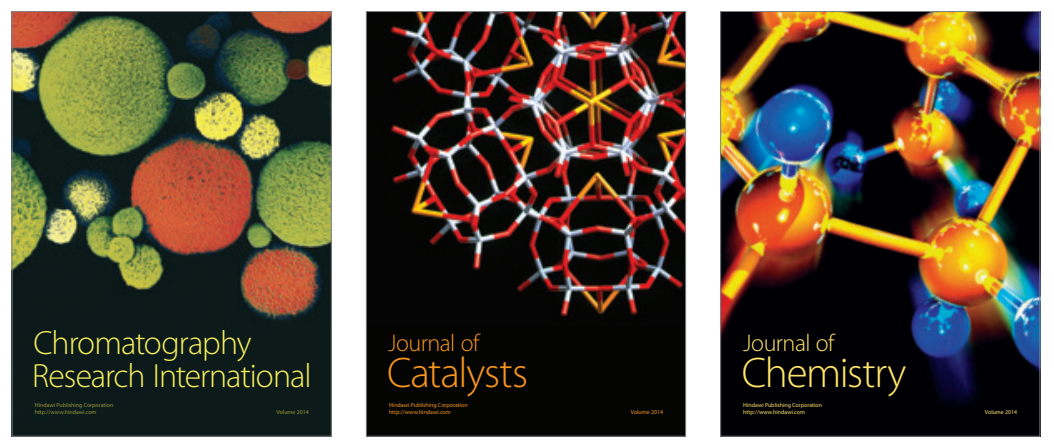
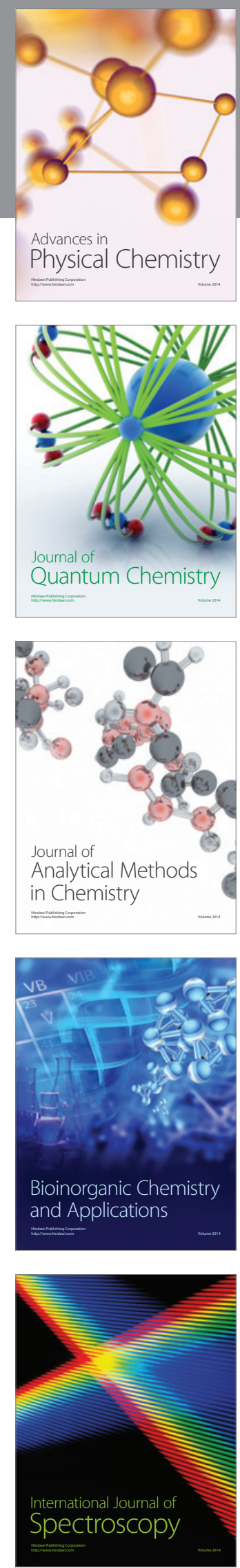